\title{
Some remarks on the particle multiplicities
}

\author{
K. Fiałkowski, R. Wit ${ }^{\mathrm{a}}$ \\ M. Smoluchowski Institute of Physics, Jagellonian University, ul. Reymonta 4, 30-059 Kraków, Poland
}

Received: 5 July 2010 / Revised: 28 August 2010 / Published online: 5 October 2010

(C) The Author(s) 2010. This article is published with open access at Springerlink.com

\begin{abstract}
Recent ALICE data for the multiplicity distributions in the central rapidity bins at LHC energies are compared with the results from two default versions of the PYTHIA 8 generator. We find that, contrary to the earlier versions of PYTHIA, the model overestimates the increase of average multiplicity with energy. Tuning two of the model parameters one obtains reasonable agreement with data. The dependence of the normalized moments of the distribution on the rapidity bin width and on energy is also qualitatively correct.
\end{abstract}

\section{Introduction}

The data on the charged particle density in the central rapidity bins were recently published by the ALICE collaboration $[1,2]$ (similar data are available from the CMS collaboration $[3,4]$, whereas the ATLAS [5] data are limited to relatively high $p_{\mathrm{T}}$ ). The energy dependence of the average multiplicity in the central unit of pseudorapidity found for the new range seems to be stronger than in the lower energy data and the observed increase is claimed to exceed significantly that expected from the PYTHIA6 [6] and PHOJET [7] event generators.

In this note we show that the situation is very different for the PYTHIA 8 generator (written in $\mathrm{C}++$ ) $[8,9]$. The default versions of this generator yield an increase of average multiplicity which is not only much stronger than that from the PYTHIA 6, but also stronger than the increase seen in the data. Tuning just two of the model parameters one obtains a good description of data. Since the PYTHIA 8 generator is still in the development stage, we use not only the most recent 8.135 version [9], but compare it with an earlier 8.107 version [8] which describes differently the diffractive processes. These versions differ in the predictions for the energy dependence of the particle density in the inelastic events. We discuss shortly the choices of event classes used

a e-mail: romuald.wit@uj.edu.pl in different analyses of the ALICE data. We compare also the model results with the data for average multiplicity and the normalized moments of the multiplicity distributions in the rapidity bins of various width for two lower LHC energies and find a reasonable agreement of the tuned version of PYTHIA 8.135 with the data.

Obviously the multiplicity distributions are not "infrared safe" as they depend significantly on the hadronization procedure and the (dis)agreement of such data with models should not be taken as a decisive test of their "validity". The tuning of MC models restricted to their description of multiplicities should be treated rather as a first step toward a proper construction of these models.

In the following section we give the details of our generation procedure and of the definitions of quantities to be compared with data. Then we present the results and compare them with the ALICE data. Short conclusions are contained in the last section.

\section{Procedures and definitions}

In this note we are using the recent $\mathrm{C}++$ versions of the PYTHIA generator: 8.107 and 8.135. Samples of 100000 minimum bias events are generated for the pp collisions at LHC energies. To discuss the influence of the changes in the diffractive component [10] we generate separately the single and double diffractive events, as well as the full samples of inelastic events.

In the ALICE data at $900 \mathrm{GeV}$ and $2.36 \mathrm{TeV}$ [1] a table of the average charged particle multiplicities is given for the CM pseudorapidity interval $|\eta|<0.5$ (i.e., it is the density in pseudorapidity). Three categories of events are considered: inelastic, non-single-diffractive and inelastic with $N_{c h}>0$. The results are compared with the old UA5 measurements and the model calculations from three models: Quark Gluon String Model, three versions of PYTHIA 6 (different "tunings") and PHOJET. There is no significant discrepancy between two sets of data, and the model results spread around 
the experimental values, although the increase with energy is systematically underestimated.

The data at $7 \mathrm{TeV}$ [2] are compared with the lower energy data and with the predictions of PYTHIA and PHOJET generators just for one class of events: inelastic with $N_{c h}>0$. Here one defines the density slightly differently: it is a half of the average multiplicity in the pseudorapidity interval $|\eta|<1$. One sees a clear discrepancy between all the models and data.

Let us note that the different definitions of the sets of data to be compared with models rely in a different degree on the models themselves. To get the "non-single-diffractive" sample one has to remove from the data the single-diffractive events, which obviously cannot be done in a model independent way. On the other hand, the "inelastic" sample is measured in the model independent way, but the model calculations treat the diffractive events in a different way than the non-diffractive ones. Thus the (dis)agreement between the models and data depends always on the description of both diffractive and non-diffractive events.

The single and double diffraction are described differently in the "old" versions of PYTHIA (PYTHIA 6 and PYTHIA 8 up to the 8.130 version) and in PYTHIA 8.135. At $7 \mathrm{TeV} \mathrm{CM}$ energy the charged multiplicity distributions for the single-diffractive component from PYTHIA 6.414 is rather sharply cut around the multiplicity of forty charged particles (in full phase space); from PYTHIA 8.135 one gets a long tail extending beyond the multiplicity of one hundred [10]. We have checked that the PYTHIA 8.107 version provides similar results as PYTHIA 6.414. An analogous effect appears for double diffraction.

Therefore the "INEL $>0$ " class contains different contributions of the diffractive events in the two versions of PYTHIA, although the total cross sections for nondiffractive, single-diffractive and double-diffractive interactions are the same for both versions at all energies. The percentage of non-diffractive events increases slowly with energy (from $65.6 \%$ to $67.6 \%$ ) and so does the percentage of inelastic events with no charge particles in the central bin. However the increase of the percentage of the "INEL $>0$ " events is faster in PYTHIA 8.135: from $81.1 \%$ to $84.7 \%$ (84.2\% in PYTHIA 8.107). This proves that there are more diffractive events in the sample for PYTHIA 8.135.

The proper definition and separation of the diffractive component is obviously crucial for the proper interpretation of the data. A more comprehensive discussion on this subject may be found in the recent paper by Khoze et al. [11].

\section{PYTHIA 8 and the ALICE data}

The most recent ALICE paper [2] presents a table of charged particle pseudorapidity density measured at central pseudorapidity $(|\eta|<1)$ for inelastic collisions having at least one
Table 1 Central density: data and the results for three versions of PYTHIA

\begin{tabular}{lllll}
\hline Energy $(\mathrm{TeV})$ & ALICE & 8.107 & 8.135 & 8.135 tuned \\
\hline 0.9 & $3.81(1)(7)$ & 3.81 & 4.00 & 3.83 \\
2.36 & $4.70(1)(11)$ & 4.93 & 5.36 & 4.67 \\
7.0 & $6.01(1)(20)$ & 6.58 & 7.66 & 5.95 \\
\hline
\end{tabular}

charged particle in the same region. The experimental data are compared with PHOJET and three versions of PYTHIA 6 generators at three LHC energies. All the generators underestimate the density with the exception of PYTHIA with ATLAS-CSC tune at $900 \mathrm{GeV}$. Moreover, all generators underestimate significantly the increase of density with energy, predicting the $15-18 \%$ increase between 900 and $2360 \mathrm{GeV}$, where the experimental increase is $23.3(+1.1 /-0.7) \%$, and the $33-48 \%$ increase between $900 \mathrm{GeV}$ and $7 \mathrm{TeV}$, where the data show a $57.6(+3.6 /-1.8) \%$ increase.

We have performed the same calculation using the default versions of PYTHIA 8.107 and 8.135 generators. The results are shown in Table 1 and in Fig. 1. We see that the PYTHIA 8.107 results agree perfectly with data at $900 \mathrm{GeV}$ and show faster increase with energy than the data: the corresponding increase between 900 and $2360 \mathrm{GeV}$ is $29.4 \%$, and between $900 \mathrm{GeV}$ and $7 \mathrm{TeV}$ it is $72.8 \%$. The disagreement is even stronger for the PYTHIA 8.135 generator: here all the values are significantly higher than the data, and the increase is $33.9 \%$ between 900 and $2360 \mathrm{GeV}$ and $91.5 \%$ between $900 \mathrm{GeV}$ and $7 \mathrm{TeV}$. Thus instead of a significant underestimation, we get now a significant overestimation of the experimental increase of the central density with energy. However, by tuning just two of the PYTHIA 8.135 parameters we are able to reproduce the experimental values from ALICE. These two parameters ecmRef and ecmPow are determining the low $p_{\mathrm{T}}$ regularization of the (divergent) $\mathrm{QCD}$ cross section by introduction of a factor

$F\left(p_{\mathrm{T}}\right)=\frac{p_{\mathrm{T}}^{4}}{\left(p_{\mathrm{T} 0}^{2}+p_{\mathrm{T}}^{2}\right)^{2}}$

where

$p_{\mathrm{T} 0}=\operatorname{pT} 0 \operatorname{Ref}\left(\frac{\text { ecmNow }}{\text { ecmRef }}\right)^{\text {ecmPow }}$

and ecmNow is the CM energy in GeV. For the pT0Ref parameter we take the default value of 2.0 , whereas ecmRef is changed from 1960.0 to 1250.0 and ecmPow from 0.16 to 0.26 . With these values of parameters we reproduce within errors the experimental values, as seen in the last column of Table 1 and in Fig. 1.

Obviously, more work is needed to tune PYTHIA 8 for the global description of LHC data. However, we may safely say that we have found a counterexample to the claim that 


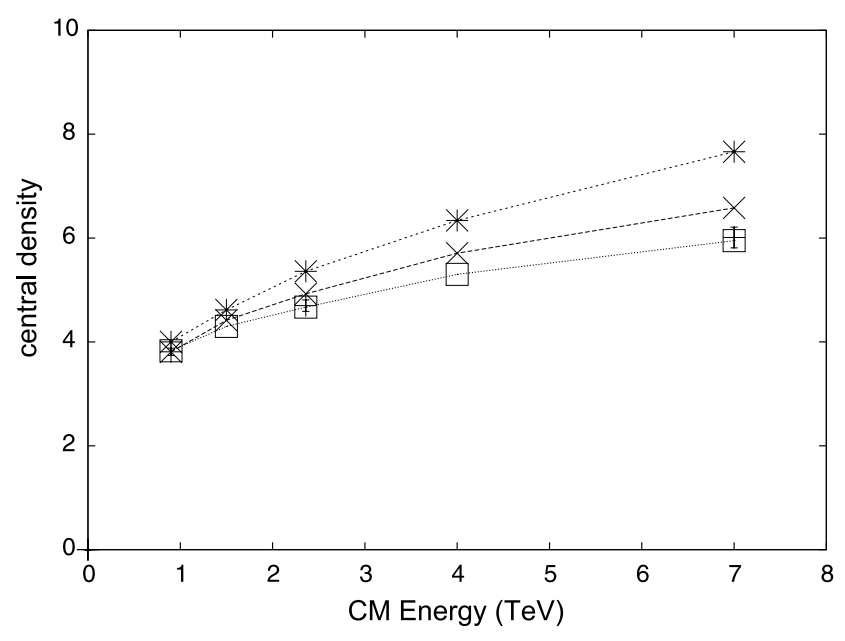

Fig. 1 The central density in pseudorapidity for the ALICE $p p$ data (crosses with error bars), PYTHIA 8.107 ( $\times$ signs), PYTHIA 8.135 default (asterisks) and PYTHIA 8.135 tuned (squares) as a function of the CM energy. The model prediction points are connected by lines to guide the eye

the increase of density with energy at LHC range is faster than expected in the commonly used generators. Moreover, since PYTHIA 8 (as well as PYTHIA 6) is based on the string fragmentation mechanism and not on the thermodynamical picture, our results suggest that it is not necessary to invoke the power-like thermodynamical increase of multiplicity with energy to describe the LHC data. Tuning the above mentioned two parameters one is able to reproduce this increase quite well.

In the first paper [1] the ALICE Collaboration has shown the data for "non-single-diffractive" events for two energies $(0.9$ and $2.360 \mathrm{TeV})$ and for three choices of the central rapidity bin width. The average multiplicities, and the three lowest normalized moments of the multiplicity distribution $c_{i}=\left\langle n^{i}\right\rangle /\langle n\rangle^{i}$ were measured. These results were shown to agree with the old UA5 data at $900 \mathrm{GeV}$; no comparison with any model was presented there.

In Tables 2 and 3 we present the comparison of these results at $0.9 \mathrm{TeV}$ and $2.36 \mathrm{TeV}$, respectively, with those from the default and tuned versions of PYTHIA 8.135.

For the default version of PYTHIA 8.135 the average multiplicity is always significantly too high. The tuned version is in perfect agreement with the data. The values of the moments are not too well reproduced, but only in one case the deviation from data is more than thrice the experimental errors. The tuning in some cases increases this deviation, and in some cases reduces it. The differences between the values from the default and tuned version of PYTHIA 8.135 may be taken as the lower limit of the uncertainties of the model; obviously, the other versions of PYTHIA may increase significantly the spread of these values. Let us stress here that the parameters were chosen to fit the data from Table 1, and not the data from Tables 2 and 3. In any case,
Table 2 Average multiplicities and moments for three choices of rapidity bin from ALICE and two versions of PYTHIA 8.135 at $0.9 \mathrm{TeV}$. The numbers in parentheses denote the statistical and systematic errors

\begin{tabular}{lllrr}
\hline Quantity & $\eta$ range & ALICE & Default & Tuned \\
\hline $\bar{n}$ & $|\eta|<0.5$ & $3.60(2)(11)$ & 4.11 & 3.72 \\
$\bar{n}$ & $|\eta|<1.0$ & $7.38(3)(17)$ & 8.35 & 7.58 \\
$\bar{n}$ & $|\eta|<1.3$ & $9.73(12)(19)$ & 10.95 & 9.93 \\
$c_{2}$ & $|\eta|<0.5$ & $1.96(1)(6)$ & 1.81 & 1.81 \\
$c_{2}$ & $|\eta|<1.0$ & $1.77(1)(4)$ & 1.66 & 1.64 \\
$c_{2}$ & $|\eta|<1.3$ & $1.70(3)(7)$ & 1.62 & 1.60 \\
$c_{3}$ & $|\eta|<0.5$ & $5.35(6)(31)$ & 4.70 & 4.75 \\
$c_{3}$ & $|\eta|<1.0$ & $4.25(3)(20)$ & 3.92 & 3.89 \\
$c_{3}$ & $|\eta|<1.3$ & $3.91(10)(15)$ & 3.71 & 3.67 \\
$c_{4}$ & $|\eta|<0.5$ & $18.3(4)(1.6)$ & 15.66 & 16.34 \\
$c_{4}$ & $|\eta|<1.0$ & $12.6(1)(9)$ & 11.76 & 11.87 \\
$c_{4}$ & $|\eta|<1.3$ & $10.9(4)(6)$ & 10.74 & 10.79 \\
\hline
\end{tabular}

Table 3 Data and models as in Table 2 at $2.36 \mathrm{TeV}$

\begin{tabular}{llcrr}
\hline Quantity & $\eta$ range & ALICE & Default & Tuned \\
\hline $\bar{n}$ & $|\eta|<0.5$ & $4.47(3)(10)$ & 5.54 & 4.55 \\
$\bar{n}$ & $|\eta|<1.0$ & $9.08(6)(29)$ & 11.25 & 9.23 \\
$\bar{n}$ & $|\eta|<1.3$ & $11.86(22)(45)$ & 14.75 & 12.09 \\
$c_{2}$ & $|\eta|<0.5$ & $2.02(1)(4)$ & 1.93 & 1.86 \\
$c_{2}$ & $|\eta|<1.0$ & $1.84(1)(6)$ & 1.81 & 1.72 \\
$c_{2}$ & $|\eta|<1.3$ & $1.79(3)(7)$ & 1.77 & 1.68 \\
$c_{3}$ & $|\eta|<0.5$ & $5.76(9)(26)$ & 5.42 & 5.07 \\
$c_{3}$ & $|\eta|<1.0$ & $4.65(6)(30)$ & 4.72 & 4.27 \\
$c_{3}$ & $|\eta|<1.3$ & $4.35(16)(33)$ & 4.51 & 4.05 \\
$c_{4}$ & $|\eta|<0.5$ & $20.6(6)(1.4)$ & 19.2 & 17.84 \\
$c_{4}$ & $|\eta|<1.0$ & $14.3(3)(1.4)$ & 15.3 & 13.53 \\
$c_{4}$ & $|\eta|<1.3$ & $12.8(7)(1.5)$ & 14.2 & 12.38 \\
\hline
\end{tabular}

the qualitative features of the data, as the decrease of moments with the width of the rapidity bin and the increase with energy, are reproduced quite well in both versions of PYTHIA. Dedicated tuning of the parameters should improve the agreement with data.

\section{Conclusions}

We have investigated the ALICE data for the multiplicity distributions in the central rapidity region using two default versions of the PYTHIA 8 generator. We find that, in comparison with data, the model overestimates the increase of central density with energy, contrary to the older versions of the PYTHIA generator. The increase is strongest for the 8.135 version, which includes the hard diffractive processes. This shows that the string models are not bound to underestimate the increase of multiplicity with energy seen at LHC 
(as often suggested). Tuning the model parameters one obtains a good agreement with data.

The data for higher moments of the multiplicity distribution for two lower LHC energies show similar features. None of the versions of the generator reproduces the data really well, but the results fall around the data. It is quite likely that a systematically tuned version may describe the experimental results in a satisfactory way.

Note added in proof: After the preparation of the final version of this note we learned of the new version of PYTHIA: PYTHIA 8.142, differing from the versions discussed by us. The reason for the changes, concerning mainly the final state radiation, was the discovered discrepancy with Tevatron data on "underlying event" (http://home.thep.lu.se/ torbjorn/ talks/CorkeTools10.pdf). We checked that the default version of PYTHIA 8.142 results in the values of central density similar to that from PYTHIA 8.107, but the increase with energy is slightly faster. All the qualitative statements presented in this note remain valid.
Acknowledgements We thank Torbjoern Sjoestrand for suggesting the choice of parameters to be tuned. We are grateful to Andrzej Kotański for helpful remarks.

Open Access This article is distributed under the terms of the Creative Commons Attribution Noncommercial License which permits any noncommercial use, distribution, and reproduction in any medium, provided the original author(s) and source are credited.

\section{References}

1. K. Aamodt et al. (ALICE Coll.), arXiv:1004.3034

2. K. Aamodt et al. (ALICE Coll.), arXiv:1004.3514

3. The CMS Collaboration, arXiv:1002.0621

4. The CMS Collaboration, arXiv:1005.3299

5. The ATLAS Collaboration, arXiv: 1003.3124

6. T. Sjöstrand, Comput. Phys. Commun. 82, 74 (1994)

7. R. Engel, J. Ranft, S. Roesler, Phys. Rev. D 52, 1459 (1995)

8. T. Sjöstrand, S. Mrenna, P. Skands, JHEP 05, 026 (2006). arXiv: 0710.3820

9. T. Sjöstrand, S. Mrenna, P. Skands, Comput. Phys. Commun. 178, 852 (2008)

10. S. Navin, arXiv:1005.3894

11. V.A. Khoze et al., Eur. Phys. J. C 69, 85 (2010) 